\title{
Numerical Solutions of Two-Way Propagation of Nonlinear Dispersive Waves Using Radial Basis Functions
}

\author{
Pablo U. Suárez ${ }^{1}$ and J. Héctor Morales ${ }^{2}$ \\ ${ }^{1}$ Department of Mathematical Sciences, Delaware State University, Dover, DE 19901, USA \\ ${ }^{2}$ Departamento de Matemáticas, UAM Iztapalapa, Apartado Postal 55-534, 09340 Iztapalapa, DF, Mexico
}

Correspondence should be addressed to Pablo U. Suárez; psuarez@desu.edu

Received 28 May 2014; Accepted 15 July 2014; Published 3 August 2014

Academic Editor: Nikolai A. Kudryashov

Copyright (C) 2014 P. U. Suárez and J. H. Morales. This is an open access article distributed under the Creative Commons Attribution License, which permits unrestricted use, distribution, and reproduction in any medium, provided the original work is properly cited.

\begin{abstract}
We obtain the numerical solution of a Boussinesq system for two-way propagation of nonlinear dispersive waves by using the meshless method, based on collocation with radial basis functions. The system of nonlinear partial differential equation is discretized in space by approximating the solution using radial basis functions. The discretization leads to a system of coupled nonlinear ordinary differential equations. The equations are then solved by using the fourth-order Runge-Kutta method. A stability analysis is provided and then the accuracy of method is tested by comparing it with the exact solitary solutions of the Boussinesq system. In addition, the conserved quantities are calculated numerically and compared to an exact solution. The numerical results show excellent agreement with the analytical solution and the calculated conserved quantities.
\end{abstract}

\section{Introduction}

Consider the initial and boundary value problem

$$
\begin{gathered}
v_{t}+u_{x}+(u v)_{x}+a u_{x x x}-b v_{x x t}=0, \\
u_{t}+v_{x}+u u_{x}+c v_{x x x}-d u_{x x t}=0, \\
\lim _{x \rightarrow \pm \infty} v(x, t)=\lim _{x \rightarrow \infty} v_{x}(x, t)=0, \\
\lim _{x \rightarrow \pm \infty} u(x, t)=\lim _{x \rightarrow \infty} u_{x}(x, t)=0, \\
v(x, 0)=v_{0}(x), \quad u(x, 0)=u_{0}(x),
\end{gathered}
$$

where $a, b, c$, and $d$ are real constants and subscripts $x$ and $t$ denote space and time derivatives, respectively. In fluid mechanics, the functions $v$ and $u$ represent flow velocities. Solutions of this type of systems have attracted much research in the past two decades [1-9]. In these studies the most popular system of Boussinesq type is the one proposed by Bona and Chen in [1], to describe approximately the two-dimensional propagation of surface waves in a uniform horizontal channel of a fixed length filled with an irrotational, incompressible, and inviscid flow. The system is derived formally from Euler's equations in 2D and using small amplitude and long wave length assumptions. Further, solitary wave solutions of this system have been reported in numerous works; see for instance $[1,2,6,10]$.

This work studies the numerical solution of this system by means of radial basis functions (RBFs). The use of these types of basis functions has become very popular in recent times; see for instance the work of Buhmann [11], Franke and Schaback [12], Driscoll and Heryudono [13], and the references therein. The main inspiration for this work is the very successful application of RBFs to solve the Kawahara equation [14] and the modified regularized long wave equation (MRLW) [15].

We will examine the two cases for the Boussinesq type system. The first one we examine is the Bona and Chen system, which is important in the theory of dispersive waves,

$$
\begin{aligned}
& v_{t}+u_{x}+(u v)_{x}-\frac{1}{6} v_{x x t}=0 \\
& u_{t}+v_{x}+u u_{x}-\frac{1}{6} u_{x x t}=0 .
\end{aligned}
$$


This corresponds to the case $a=c=0$ with $b=d=1 / 6$. The second case we examine is the full system with $a=-7 / 30$, $b=7 / 15, c=-2 / 5$, and $d=1 / 2$ :

$$
\begin{gathered}
v_{t}+u_{x}+(v u)_{x}-\frac{7}{30} u_{x x x}-\frac{7}{15} v_{x x t}=0 \\
u_{t}+v_{x}+u u_{x}-\frac{2}{5} v_{x x x}-\frac{1}{2} u_{x x t}=0 .
\end{gathered}
$$

The paper is organized in three sections. In Section 2, we use RBFs to reduce the PDE system to a system of differential equations. In Section 3, we use a linear multistep method to solve these ODEs and then proceed to compute several conserved quantities of the PDE system. We provide comparative tables in order to show the accuracy of the RBFs approach. Finally, in Section 4, we discuss some conclusions.

\section{The Method of Lines for Radial Basis Functions}

The RBFs approach is one of the most effective meshless methods to solve numerically PDEs. The key feature of the RBFs is that its implementation does not require a mesh at all. The method employs approximants whose values depend only on the distance between some center point and a domain point, in an appropriate norm. Due to the use of the distance functions, we can control the convergence up to an order proportional to the spacing of two points where we evaluate such functions [16].

The functions $v(x, t)$ and $u(x, t)$ in (1) and (2) are approximated using $n$-terms RBFs:

$$
\begin{aligned}
& v(x, t) \approx v_{n}(x, t)=\sum_{j=0}^{n} \phi_{j}(x) \lambda_{j}(t), \\
& u(x, t) \approx u_{n}(x, t)=\sum_{j=0}^{n} \phi_{j}(x) \mu_{j}(t),
\end{aligned}
$$

where $\lambda_{j}(t)$ and $\mu_{j}(t)$ are time-dependent functions to be determined and $\phi_{j}(x):=\phi\left(\left\|x-x_{j}\right\|\right)$ is a radial basis functions to be specified. We adopt the Euclidean $L_{2}$-norm $\|\cdot\|_{2}$ in this work to denote distance between the point $x_{j}$, referred as center of the RBFs, and $x$, the free spatial variable, at which we can evaluate the approximant. Some examples of the RBFs are $[11,15]$

$$
\begin{gathered}
\text { Multi-quadratic(MQ) } \phi_{j}(x)=\left(\left(x-x_{j}\right)^{2}+\varphi^{2}\right)^{1 / 2}, \\
\text { Gaussian (G) } \phi_{j}(x)=e^{-\varphi^{2}\left(x-x_{j}\right)^{2}}, \\
\text { Inverse-quadratic(IQ) } \phi_{j}(x)=\left(\left(x-x_{j}\right)^{2}+\varphi^{2}\right)^{-1},
\end{gathered}
$$

Inverse Multi-quadratic (IMQ)

$$
\phi_{j}(x)=\left(\left(x-x_{j}\right)^{2}+\varphi^{2}\right)^{-1 / 2}
$$

where the parameter $\varphi$ is proportional to the amplitude of the $\mathrm{RBF}$ at $x=x_{j}$, and it is under our control [11]. These RBFs have shown to be successful in solving nonlinear PDEs with mixed partial derivatives $[15,17]$.

In terms of the RBFs, we can find the derivatives of the approximate solution of $n$ terms as follows:

$$
\begin{aligned}
\frac{\partial^{k}}{\partial x^{k}} v_{n}(x, t) & =\sum_{j=0}^{n} \phi_{j}^{(k)}(x) \lambda_{j}(t), \\
\frac{\partial}{\partial t} v_{n}(x, t) & =\sum_{j=0}^{n} \phi_{j}(x) \dot{\lambda}_{j}(t),
\end{aligned}
$$

as well as for $u_{n}$.

If we substitute (8)-(11) into (1)-(2), we obtain a system of two coupled nonlinear ordinary differential equations for $\lambda_{j}(t)$ and $\mu_{j}(t)$ :

$$
\begin{aligned}
\sum_{j=0}^{n}\left[\left(\phi_{j}(x)-b \phi_{j}^{(2)}(x)\right) \dot{\lambda}_{j}(t)+\left(\phi_{j}^{(1)}(x)+a \phi_{j}^{(2)}(x)\right) \mu_{j}(t)\right] \\
=-\sum_{j=0}^{n} \phi_{j}^{(1)}(x) \mu_{j}(t) \sum_{j=0}^{n} \phi_{j}(x) \lambda_{j}(t) \\
\quad-\sum_{j=0}^{n} \phi_{j}(x) \mu_{j}(t) \sum_{j=0}^{n} \phi_{j}^{(1)}(x) \lambda_{j}(t),
\end{aligned}
$$$$
\sum_{j=0}^{n}\left[\phi_{j}(x) \dot{\lambda}_{j}(t)+\left(\phi_{j}^{(1)}(x)+c \phi_{j}^{(3)}(x)\right) \lambda_{j}(t)\right.
$$$$
\left.-d \phi_{j}^{(2)}(x) \dot{\mu}_{j}(t)\right]=-\sum_{j=0}^{n} \phi_{j}(x) \mu_{j}(t) \sum_{j=0}^{n} \phi_{j}^{(1)}(x) \mu_{j}(t),
$$

where $\phi_{j}(x)=\phi\left(\left\|x-x_{j}\right\|\right)$.

We solve the system over the real line $-\infty \leq x \leq \infty$ and on a finite time $0 \leq t \leq \tau$. We first truncate the spatial interval to a finite computational domain $-L \leq x \leq L$ and specify $m+1$ collocation points $x_{0}, x_{1}, \ldots, x_{m}$. We then employ these equally-spaced collocation points $x_{i}=-L+i \Delta x, i=0, \ldots, m$; $\Delta x=2 L / m$ and introduce the matrix notation

$$
\begin{aligned}
(\Phi)_{i j} & \left.\equiv \phi_{j}(x)\right|_{x=x_{i}} \in \mathbb{R}^{(m-1)} \times \mathbb{R}^{(n-1)}, \\
\left(D_{k} \Phi\right)_{i j} & \left.\equiv\left(\frac{\partial^{k} \Phi}{\partial x^{k}}\right)\right|_{x=x_{i}} \in \mathbb{R}^{(m-1)} \times \mathbb{R}^{(n-1)}, \\
\lambda(t) & \equiv\left(\lambda_{1}(t), \lambda_{2}(t), \ldots, \lambda_{n}(t)\right)^{T} \in \mathbb{R}^{(n-1)}, \\
\boldsymbol{\mu}(t) & \equiv\left(\mu_{1}(t), \mu_{2}(t), \ldots, \mu_{n}(t)\right)^{T} \in \mathbb{R}^{(n-1)} ;
\end{aligned}
$$

the vector superscript $T$ denotes the transpose. We then obtain a $2(m-1) \times 2(n-1)$ ODE system that can be compactly written in the form

$$
M \dot{\mathbf{y}}(t)+\Gamma \mathbf{y}(t)=F(t, \mathbf{y}(t))
$$

where $M$ is called a mass matrix, $\Gamma$ is called the drift matrix, and $F$ is in general a nonlinear term dependent on $t$ and $\mathbf{y}(t)$. 
The vector $\mathbf{y}(t)$ is defined by $\mathbf{y}(t)=[\boldsymbol{\lambda}(t), \boldsymbol{\mu}(t)]^{T}$. Explicitly, we have

$$
\begin{aligned}
M & =\left[\begin{array}{cc}
\left(1-b D_{x x}\right) \boldsymbol{\Phi} & \mathbf{0} \\
\mathbf{\Phi} & -d D_{x x} \boldsymbol{\Phi}
\end{array}\right]_{2(m-1) \times 2(n-1)}, \\
\Gamma & =\left[\begin{array}{cc}
\mathbf{0} & -\left(D_{x}+a D_{x x}\right) \boldsymbol{\Phi} \\
\left(D_{x}+c D_{x x x}\right) \boldsymbol{\Phi} & \mathbf{0}
\end{array}\right]_{2(m-1) \times 2(n-1)}, \\
F & =\left[\begin{array}{c}
-D_{x}(\boldsymbol{\Phi} \boldsymbol{\mu} \boldsymbol{\Phi} \boldsymbol{\lambda}) \\
-\boldsymbol{\Phi} \boldsymbol{\mu}\left(D_{x} \boldsymbol{\Phi}\right) \boldsymbol{\mu}
\end{array}\right]_{2(m-1) \times 1} .
\end{aligned}
$$

The elements of $F$ must be explained. The spatial derivative (15), $D_{k}$, with $k=x, x x$ or $x x x$, is acting element by element on a matrix and does not modify its size. The products $\Phi \boldsymbol{\mu}$ and $\boldsymbol{\Phi} \boldsymbol{\lambda}$ are vectors of size $(m-1) \times 1$ each; however, its product is element by element according to the righthand side of (12), after the expression is evaluated in a particular collocation point $x_{i}$. Therefore, $-D_{x}(\Phi \boldsymbol{\mu} \Phi \boldsymbol{\lambda})$ and $-\boldsymbol{\Phi} \boldsymbol{\mu}\left(D_{x} \boldsymbol{\Phi}\right) \boldsymbol{\mu}$ are vectors of size $(m-1) \times 1$.

Additionally, the discretization of the boundary conditions, equations (3) and (4), provide four algebraic equations:

$$
\begin{gathered}
v_{n}\left(t, x_{0}\right)=v_{n}\left(t, x_{m}\right)=\sum_{j=0}^{n} \phi_{j}\left(x_{m}\right) \lambda_{j}(t)=0, \\
\text { at } x=-L, L, \\
u_{n}\left(t, x_{0}\right)=u_{n}\left(t, x_{m}\right)=\sum_{j=0}^{n} \phi_{j}\left(x_{m}\right) \mu_{j}(t)=0, \\
\text { at } x=-L, L, \\
v_{n}^{(1)}\left(t, x_{m}\right)=\sum_{j=0}^{n} \phi_{j}^{(1)}\left(x_{m}\right) \lambda_{j}(t)=0, \\
\text { at } x=L, \\
u_{n}^{(1)}\left(t, x_{m}\right)=\sum_{j=0}^{n} \phi_{j}^{(1)}\left(x_{m}\right) \mu_{j}(t)=0, \\
\text { at } x=L,
\end{gathered}
$$

and the initial conditions (5) provide us with two algebraic equations:

$$
\begin{aligned}
v_{n}(0, x)=\sum_{j=0}^{n} \phi_{j}(x) \lambda_{j}(0) & =v_{0}(x), \\
(-L & \leq x \leq L), \\
u_{n}(0, x)=\sum_{j=0}^{n} \phi_{j}(x) \mu_{j}(0) & =u_{0}(x), \\
(-L & \leq x \leq L) .
\end{aligned}
$$

To solve the system (18), we use the fourth-order RungeKutta Method (RK4) and let $m=n$. We can rewrite (18) as

$$
\dot{\mathbf{y}}=\mathbf{f}(\mathbf{y}, t),
$$

where $\mathbf{f}(\mathbf{y}, t)=M^{-1}(-\Gamma \mathbf{y}(t)+F(t, \mathbf{y}(t)))$.

The classical RK4 algorithm for a system of ODEs is given by

$$
\mathbf{y}^{(n+1)}=\mathbf{y}^{(n)}+\frac{\Delta t}{6}\left(\mathbf{k}_{1}+2 \mathbf{k}_{2}+2 \mathbf{k}_{3}+\mathbf{k}_{4}\right)
$$

where

$$
\begin{aligned}
& \mathbf{k}_{1}=\mathbf{f}\left(\mathbf{y}^{(n)}, t^{(n)}\right), \\
& \mathbf{k}_{2}=\mathbf{f}\left(\mathbf{y}^{(n)}+\frac{\Delta t}{2} \mathbf{k}_{1}, t^{(n)}+\frac{\Delta t}{2}\right), \\
& \mathbf{k}_{3}=\mathbf{f}\left(\mathbf{y}^{(n)}+\frac{\Delta t}{2} \mathbf{k}_{2}, t^{(n)}+\frac{\Delta t}{2}\right), \\
& \mathbf{k}_{4}=\mathbf{f}\left(\mathbf{y}^{(n)}+\Delta t \mathbf{k}_{3}, t^{(n)}+\Delta t\right) .
\end{aligned}
$$

The RK4 scheme does not have stability issues as long as the time step $\Delta t$ is chosen sufficiently small. The selection of the time step is done by the following [18].

Rule of Thumbs. The method of lines is stable if the eigenvalues of the linearized spatial discretization operator, scaled by $\Delta t$, lie in the stability region of the time-discretization operator.

For the stability analysis nonlinearity in $(22), F(t, \mathbf{y}(t))$ is set to zero and we compute the eigenvalues of $M^{-1} \Gamma$. So the method is stable as long as the eigenvalues are in the stability region $\left(-2.76<\Delta t \lambda_{j}<0\right)$. In order to have a stable scheme, we adjust the parameter $\varphi$ so that the matrices remain in the region of stability. This was carried out in the numerical experiments.

\section{Numerical Approximations of Boussinesq System}

In this section, we use the proposed algorithm to calculate the numerical solution of the Boussinesq type system and its conserved quantities. These quantities were performed by means of the trapezoidal quadrature. The accuracy of the method is tested by computing the $L_{2}$ and $L_{\infty}$ (error norms) defined as

$$
\begin{gathered}
L_{2}=\sqrt{\Delta x \sum_{j=0}^{N}\left|w_{j}^{e}-w_{j}^{n}\right|^{2}}, \\
L_{\infty}=\max _{j}\left|w_{j}^{e}-w_{j}^{n}\right|,
\end{gathered}
$$

where $w_{j}^{e}$ and $w_{j}^{n}$ stand for numerical evaluation of the exact solution and numerical approximation of the solution, respectively. The test cases in this section are solved over the domain $-50 \leq x \leq 50$ in the time period $0<t \leq 20$. The domain and time period are discretized with values $\Delta x=0.25$ 
TABLE 1: Error norms of $v(x, 15)$.

\begin{tabular}{|c|c|c|c|c|c|}
\hline Method & $\varphi$ & $L_{2}$ error of $v$ & $L_{\infty}$ error of $v$ & Amplitude $v$ & $x$ \\
\hline Analytic & - & - & - & 7.462309 & 37.43 \\
\hline MQ & 1.23 & $3.074 E-05$ & $2.721 E-05$ & 7.462316 & 37.43 \\
\hline G & 2.66 & $5.395 E-05$ & $4.783 E-05$ & 7.462310 & 37.43 \\
\hline IQ & 1.00 & $2.244 E-03$ & $2.129 E-03$ & 7.462390 & 37.43 \\
\hline IMQ & 2.05 & $2.450 E-03$ & $1.224 E-03$ & 7.462565 & 37.43 \\
\hline
\end{tabular}

TABLE 2: Error norms of $u(x, 15)$.

\begin{tabular}{lccccr}
\hline Method & $\varphi$ & $L_{2}$ error of $u$ & $L_{\infty}$ error of $u$ & Amplitude $(\mathrm{P}-\mathrm{P}) u$ & $x_{\min }$ \\
\hline Analytic & - & - & - & 4.91342 & 36.23 \\
MQ & 1.23 & $3.381 E-05$ & $2.606 E-05$ & 4.91343 & 37.43 \\
G & 2.66 & $5.747 E-05$ & $5.358 E-05$ & 4.91339 & 36.23 \\
IQ & 1.00 & $2.313 E-03$ & $2.204 E-03$ & 4.91575 & 36.23 \\
IMQ & 2.05 & $2.804 E-03$ & $1.950 E-03$ & 4.91481 & 37.43 \\
\hline
\end{tabular}

and $\Delta t=0.2$, respectively. The initial conditions are taken from the exact solution at the value $t=0$.

The integrals involving the conserved quantities were calculated by using the Trapezoidal rule.

3.1. Numerical Benchmark. The first test problem was studied extensively by Chen in [2]. This case is important in the theory dispersive waves. A solitary wave solutions is known and is given by Chen in [2]:

$$
\begin{aligned}
v(x, t)= & 3 k \operatorname{sech}^{2}\left(\frac{3}{\sqrt{10}}(x-k t)\right), \quad k= \pm \frac{5}{2}, \\
u(x, t)= & \frac{15}{4}\left[-2+\cosh \left(3 \sqrt{\frac{2}{5}}(x-k t)\right)\right] \\
& \times \operatorname{sech}^{4}\left(\frac{3}{\sqrt{10}}(x-k t)\right) .
\end{aligned}
$$

This case has four conserved quantities derived in [4]:

$$
\begin{aligned}
& I_{1}=\int_{-\infty}^{\infty} v d x, \\
& I_{2}=\int_{-\infty}^{\infty} u d x, \\
& I_{3}=\int_{-\infty}^{\infty}\left[v^{2}+(1+v) u^{2}\right] d x, \\
& I_{4}=\int_{-\infty}^{\infty}\left[v u+\frac{1}{6} v_{x} u_{x}\right] d x .
\end{aligned}
$$

In Tables 1 and 2 we have the error norms at time $t=$ 15 for $v(x, t)$ and $u(x, t)$, respectively. We also measure the amplitude for $v(x, t)$ and the peak-to-peak (P-P) amplitude for $u(x, t)$. Table 3 shows the analytical and numerical value of the conserved quantities. The tables show excellent agreement with the exact solution. We can see that using multiquadratic and Gaussian quadratic RBFs is more accurate in approximating the solution which is in agreement with the work of Dereli [15] for MRLW equation. In addition to these tables, we see in Figure 1 how the approximate solution is "lying" closely to the analytical one. Figure 2 shows that as we increase the number of collocation points the error reduces.

3.2. Problem. In this example we test our algorithm with a solution against the "full system" equations (7). A solitary wave solution to the "full system" is presented in [7]. The solution is as follows:

$$
\begin{gathered}
v(x, t)=\frac{3}{8} \operatorname{sech}^{2}\left(\frac{1}{2} \sqrt{\frac{5}{7}}\left[x+\frac{5 \sqrt{2}}{6} t\right]\right), \\
u(x, t)=\frac{1}{2 \sqrt{2}} \operatorname{sech}^{2}\left(\frac{1}{2} \sqrt{\frac{5}{7}}\left[x+\frac{5 \sqrt{2}}{6} t\right]\right) .
\end{gathered}
$$

The boundary conditions are the same as the BBM and the conserved quantities are

$$
\begin{aligned}
& I_{1}=\int_{-\infty}^{\infty} v d x, \\
& I_{2}=\int_{-\infty}^{\infty} u d x .
\end{aligned}
$$

Tables $4,5,6$, and 7 show the $L_{2}$ and $L_{\infty}$ error for various times of $v(x, t)$ and $u(x, t)$ along with the conserved quantities 
TABle 3: Conserved quantities.

\begin{tabular}{lcccc}
\hline Method & $I_{1}$ & $I_{2}$ & $I_{3}$ & $I_{4}$ \\
\hline Analytic & 15.81139 & 0.0000 & 158.113 & -22.5876975726 \\
MQ & 15.81134 & $-2.07 E-05$ & 158.113 & -22.58776106781 \\
G & 15.81139 & $-1.31 E-09$ & 158.113 & -22.58776062211 \\
IQ & 15.81144 & $-2.87 E-04$ & 158.168 & -22.58432200892 \\
IMQ & 15.81156 & $6.92 E-04$ & 158.152 & -22.58512792633 \\
\hline
\end{tabular}

TABLE 4: Error norms and conserved quantities for $v(x, t)$ and $u(x, t)$.

\begin{tabular}{|c|c|c|c|c|c|c|}
\hline Time & $L_{2}$ error of $v$ & $L_{\infty}$ error of $v$ & $L_{2}$ error of $u$ & $L_{\infty}$ error of $u$ & $I_{1}$ & $I_{2}$ \\
\hline \multicolumn{7}{|l|}{ MQ } \\
\hline 0.5 & $3.467 E-08$ & $4.122 E-08$ & $4.437 E-08$ & $5.202 E-08$ & 1.7748239 & 1.6733200 \\
\hline 1.0 & $1.544 E-07$ & $1.766 E-07$ & $1.892 E-07$ & $2.231 E-07$ & 1.7748238 & 1.6733198 \\
\hline 2.0 & $3.061 E-07$ & $3.130 E-07$ & $3.372 E-07$ & $3.550 E-07$ & 1.7748235 & 1.6733196 \\
\hline 3.0 & $3.960 E-07$ & $3.480 E-07$ & $4.206 E-07$ & $3.796 E-07$ & 1.7748233 & 1.6733193 \\
\hline 4.0 & $4.585 E-07$ & $3.600 E-07$ & $4.762 E-07$ & $3.825 E-07$ & 1.7748231 & 1.6733195 \\
\hline 5.0 & $4.501 E-07$ & $3.490 E-07$ & $4.604 E-07$ & $3.689 E-07$ & 1.7748232 & 1.6733192 \\
\hline 10.0 & $4.758 E-07$ & $2.765 E-07$ & $4.881 E-07$ & $2.864 E-07$ & 1.7748237 & 1.6733193 \\
\hline 15.0 & $5.607 E-07$ & $2.351 E-07$ & $5.715 E-07$ & $2.400 E-07$ & 1.7748246 & 1.6733198 \\
\hline 20.0 & $7.205 E-07$ & $2.102 E-07$ & $7.300 E-07$ & $2.138 E-07$ & 1.7748260 & 1.6733205 \\
\hline 25.0 & $1.116 E-06$ & $3.704 E-07$ & $1.143 E-07$ & $3880 E-07$ & 1.7748285 & 1.6733212 \\
\hline 30.0 & $4.239 E-06$ & $6.321 E-06$ & $4.954 E-06$ & $5.960 E-06$ & 1.7748236 & 1.6733103 \\
\hline
\end{tabular}

TABLE 5: Error norms and conserved quantities for $v(x, t)$ and $u(x, t)$.

\begin{tabular}{rrrrrr}
\hline Time & $L_{2}$ error of $v$ & $L_{\infty}$ error of $v$ & $L_{2}$ error of $u$ & $L_{\infty}$ error of $u$ & $I_{1}$ \\
\hline G & & & & & \\
0.5 & $2.268 E-10$ & $1.442 E-10$ & $2.188 E-10$ & $1.425 E-10$ & 1.7748239 \\
1.0 & $4.548 E-10$ & $2.948 E-10$ & $4.351 E-10$ & $2.877 E-10$ & 1.7748238 \\
2.0 & $9.045 E-10$ & $5.976 E-10$ & $8.672 E-10$ & $5.848 E-10$ & 1.7748235 \\
3.0 & $1.343 E-09$ & $9.038 E-10$ & $1.295 E-09$ & $8.901 E-10$ & 1.7748233 \\
4.0 & $1.775 E-09$ & $1.195 E-09$ & $1.710 E-09$ & $1.172 E-09$ & 1.7748231 \\
5.0 & $2.194 E-09$ & $1.476 E-09$ & $2.113 E-09$ & $1.445 E-09$ & 1.7748232 \\
10.0 & $4.065 E-09$ & $2.570 E-09$ & $3.909 E-09$ & $2.490 E-09$ & 1.7748237 \\
15.0 & $5.600 E-09$ & $3.353 E-09$ & $5.371 E-09$ & $3.232 E-09$ & 1.7748246 \\
20.0 & $6.900 E-09$ & $3.976 E-09$ & $6.601 E-09$ & $2.821 E-09$ & 1.7748260 \\
25.0 & $2.992 E-08$ & $4.345 E-08$ & $2.668 E-08$ & $4.097 E-08$ & 1.6733196 \\
30.0 & $4.193 E-06$ & $6.322 E-06$ & $3.718 E-06$ & $5.960 E-06$ & 1.6733193193 \\
\hline
\end{tabular}

TABLE 6: Error norms and conserved quantities for $v(x, t)$ and $u(x, t)$.

\begin{tabular}{cccccc}
\hline Time & $L_{2}$ error of $v$ & $L_{\infty}$ error of $v$ & $L_{2}$ error of $u$ & $L_{\infty}$ error of $u$ & $I_{1}$ \\
\hline IQ & & & & & \\
0.5 & $3.598 E-07$ & $4.300 E-07$ & $4.310 E-07$ & $5.425 E-07$ & 1.7748239 \\
1.0 & $2.492 E-06$ & $2.925 E-06$ & $3.060 E-06$ & $3.647 E-06$ & 1.7748238 \\
2.0 & $1.299 E-05$ & $1.268 E-05$ & $1.510 E-05$ & $1.495 E-05$ & 1.7748235 \\
3.0 & $2.101 E-05$ & $1.786 E-05$ & $2.285 E-05$ & $1.978 E-05$ & 1.7748233 \\
4.0 & $2.366 E-05$ & $1.894 E-05$ & $2.456 E-05$ & $2.006 E-05$ & 1.7748231 \\
5.0 & $3.522 E-05$ & $2.862 E-05$ & $4.034 E-05$ & $3.483 E-05$ & 1.7748232 \\
10.0 & $6.753 E-05$ & $4.177 E-05$ & $7.152 E-05$ & $4.450 E-05$ & 1.7748237 \\
15.0 & $1.342 E-04$ & $7.717 E-05$ & $1.386 E-04$ & $8.006 E-05$ & 1.77338200 \\
20.0 & $1.559 E-04$ & $7.227 E-05$ & $1.600 E-04$ & $7.515 E-05$ & 1.6733196 \\
25.0 & $2.088 E-04$ & $8.579 E-05$ & $2.150 E-04$ & $8.807 E-05$ & 1.6733193 \\
30.0 & $2.743 E-04$ & $9.665 E-05$ & $2.793 E-04$ & $9.855 E-05$ & 1.6733195 \\
\hline
\end{tabular}


TABLE 7: Error norms and conserved quantities for $v(x, t)$ and $u(x, t)$.

\begin{tabular}{rrrrrr}
\hline Time & $L_{2}$ error of $v$ & $L_{\infty}$ error of $v$ & $L_{2}$ error of $u$ & $L_{\infty}$ error of $u$ & $I_{1}$ \\
\hline IMQ & & & & & \\
0.5 & $2.081 E-05$ & $2.670 E-05$ & $2.595 E-05$ & $3.369 E-05$ & 1.7748239 \\
1.0 & $9.158 E-05$ & $1.056 E-04$ & $1.114 E-04$ & $1.310 E-04$ & 1.7748238 \\
2.0 & $2.616 E-04$ & $2.468 E-04$ & $2.967 E-04$ & $2.830 E-04$ & 1.7748235 \\
3.0 & $3.887 E-04$ & $3.171 E-04$ & $4.191 E-04$ & $3.437 E-04$ & 1.7748233 \\
4.0 & $4.852 E-04$ & $3.515 E-04$ & $5.094 E-04$ & $3.711 E-04$ & 1.7748231 \\
5.0 & $5.616 E-04$ & $3.659 E-04$ & $5.816 E-04$ & $3.815 E-04$ & 1.7748232 \\
10.0 & $8.109 E-04$ & $3.712 E-04$ & $8.225 E-04$ & $3.799 E-04$ & 1.7748237 \\
15.0 & $1.221 E-03$ & $5.494 E-04$ & $1.253 E-03$ & $5.849 E-04$ & 1.7748246 \\
20.0 & $1.783 E-03$ & $6.396 E-04$ & $1.808 E-03$ & $6.490 E-04$ & 1.77482600 \\
25.0 & $2.274 E-03$ & $6.435 E-04$ & $2.298 E-03$ & $6.496 E-04$ & 1.7733196 \\
30.0 & $2.803 E-03$ & $7.035 E-04$ & $2.835 E-03$ & $7.170 E-04$ & 1.6733193 \\
\hline
\end{tabular}
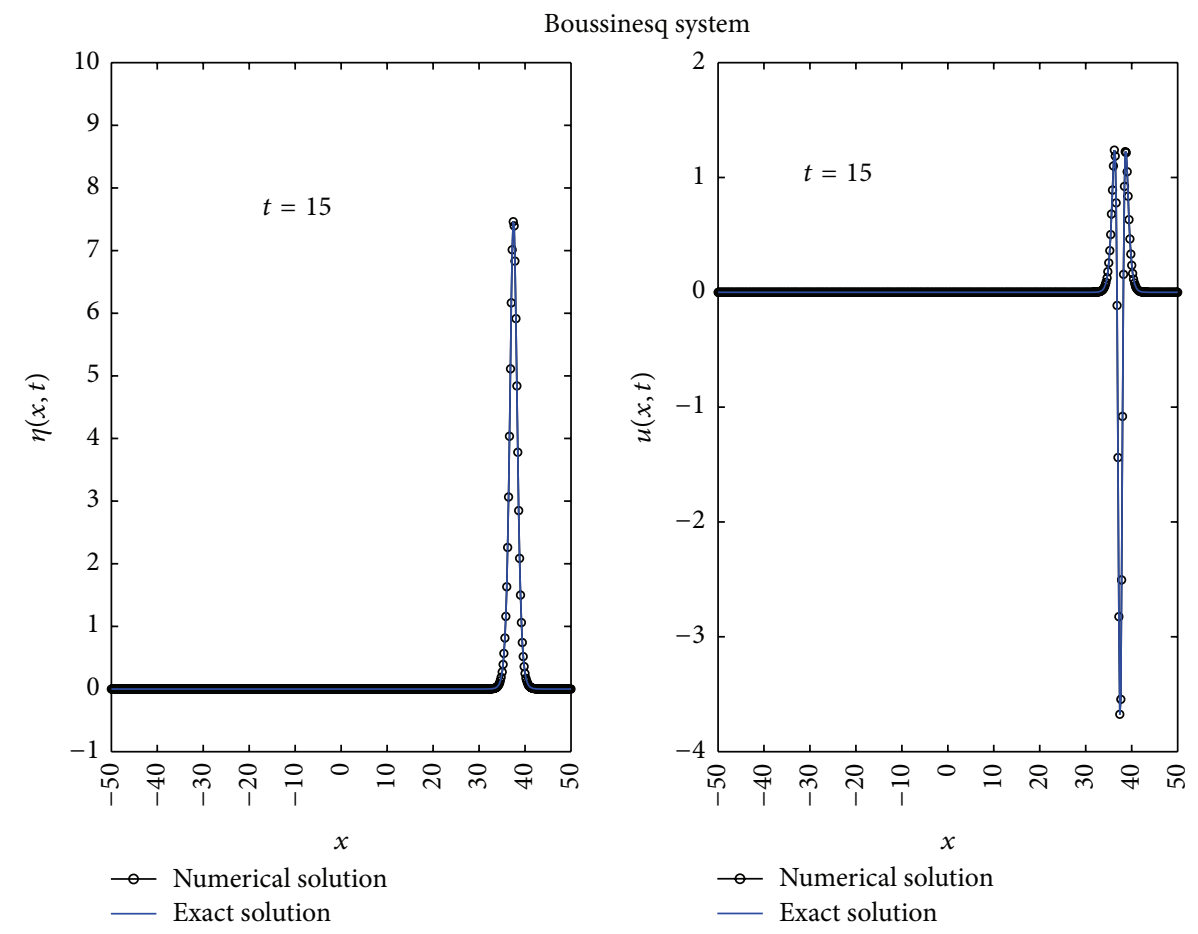

FIGURE 1: Comparison of $v(x, t)$ and $u(x, t)$ with exact solution using MQ RBFs.

of the equations. Once again the numerical solution and the analytical solution are in good agreement.

3.3. Problem. In this last example, we present the interaction between two solitons. The initial conditions are taken to be

$$
\begin{aligned}
v(x, 0)= & \frac{3}{8} \operatorname{sech}^{2}\left(\frac{1}{2} \sqrt{\frac{5}{7}}(x-10)\right) \\
& +\frac{3}{8} \operatorname{sech}^{2}\left(\frac{1}{2} \sqrt{\frac{5}{7}}(x+10)\right),
\end{aligned}
$$

$$
\begin{aligned}
u(x, 0)= & -\frac{1}{2 \sqrt{2}} \operatorname{sech}^{2}\left(\frac{1}{2} \sqrt{\frac{5}{7}}(x-10)\right) \\
& -\frac{1}{2 \sqrt{2}} \operatorname{sech}^{2}\left(\frac{1}{2} \sqrt{\frac{5}{7}}(x+10)\right) .
\end{aligned}
$$

The simulation explored in this section cannot be benchmarked via an exact analytical solution; to the authors knowledge no exact interacting soliton solution is known. However, the results are consistent with known properties of solitary waves [19]:

(1) Solitons are permanent in form.

(2) Solitons are localized within a region. 


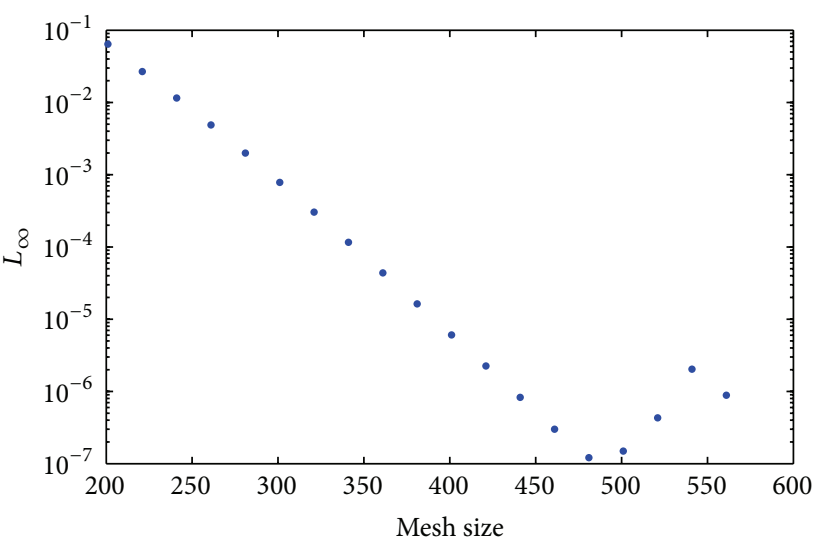

(a) Evolution of $v(x, t)$

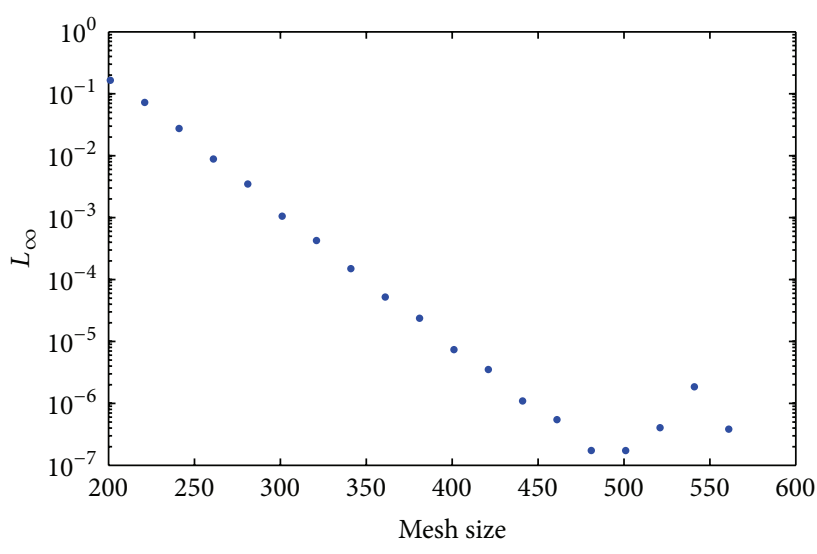

(b) Evolution of $u(x, t)$

FIGURE 2: Soliton interaction of Boussinesq type system.

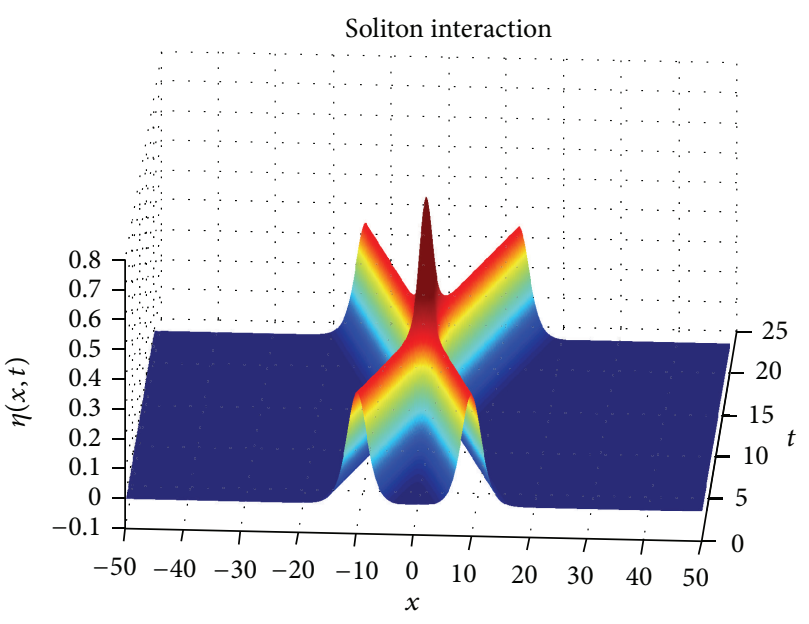

(a) $v(x, t)$

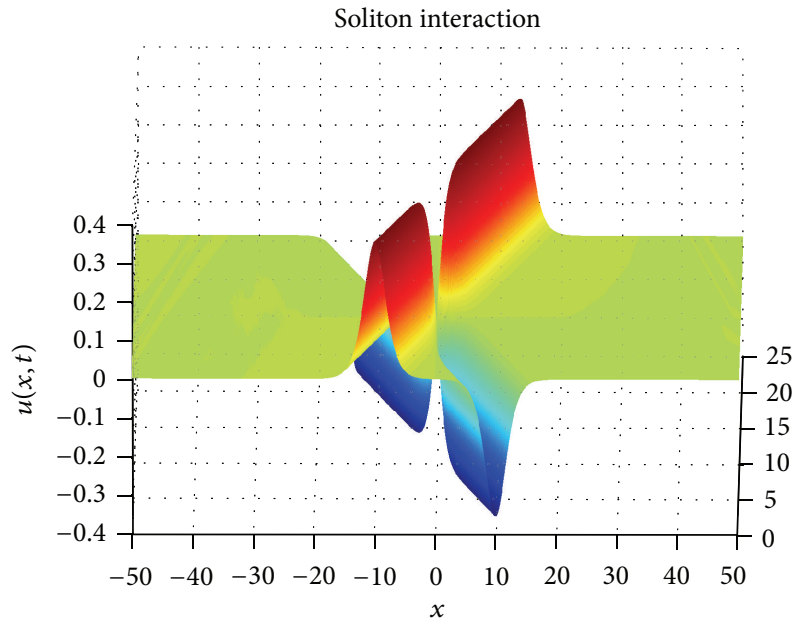

(b) $u(x, t)$

FIGURE 3: Convergence of mesh error.

(3) Solitons can interact with other solitons and emerge from the collision unchanged, except for a phase shift.

The simulation is done by using only multiquadratic radial basis functions with 200 node points in $x \in[-50,50]$. The numerical results are shown in Figures 2(a) and 2(b). We can see in both figures that the solitons remain permanent in form except at the time interval of collision. In Figure 3(a) we observe constructive interference of the solitons $v(x, t)$ at $t=8.5$. On the other hand, in Figure 3(b) we can see the colliding solitons $u(x, t)$ form destructive interference at $t=8.5$. Finally, the figures show that after the collision the solitons emerged unchanged.

\section{Conclusions}

We have solved numerically the Boussinesq type system of nonlinear PDE by means of RBFs. In general this algorithm solves the system accurately. In particular, this method has optimal results for multiquadric and Gaussian radial basis functions. The conserved quantities were calculated and show good agreement with the analytical results. In the final section we ran a simulation for interacting solitons that is in agreement with the known properties of solitons. In conclusion the meshless method is shown to give good results for a system of PDEs having high order derivatives and mixed-partial derivatives.

\section{Conflict of Interests}

The authors declare that there is no conflict of interests regarding the publication of this paper.

\section{References}

[1] J. L. Bona and M. Chen, "A Boussinesq system for twoway propagation of nonlinear dispersive waves," Physica D: Nonlinear Phenomena, vol. 116, no. 1-2, pp. 191-224, 1998.

[2] M. Chen, "Exact solutions of various Boussinesq systems," Applied Mathematics Letters, vol. 11, no. 5, pp. 45-49, 1998. 
[3] M. Chen, "Exact traveling-wave solutions to bidirectional wave equations," International Journal of Theoretical Physics, vol. 37, no. 5, pp. 1547-1567, 1998.

[4] A. A. Alazman, J. P. Albert, J. L. Bona, M. Chen, and J. Wu, "Comparisons between the BBM equation and a Boussinesq system," Advances in Differential Equations, vol. 11, no. 2, pp. 121166, 2006.

[5] J. L. Bona, M. Chen, and J.-C. Saut, "Boussinesq equations and other systems for small-amplitude long waves in nonlinear dispersive media. I. Derivation and linear theory," Journal of Nonlinear Science, vol. 12, no. 4, pp. 283-318, 2002.

[6] T. Banachiewicz, "Zur Berechnung der Determinanten, wie auch der Inversen, and zur darauf basierten Auflösung der Systeme lineare Gleichungen," Acta Astronomica C, vol. 3, pp. 41-67, 1937.

[7] J. L. Bona, M. Chen, and J.-C. Saut, "Boussinesq equations and other systems for small-amplitude long waves in nonlinear dispersive media: II. The nonlinear theory," Nonlinearity, vol. 17, no. 3, pp. 925-952, 2004.

[8] H. Chen, M. Chen, and N. V. Nguyen, "Cnoidal wave solutions to Boussinesq systems," Nonlinearity, vol. 20, no. 6, pp. 14431461, 2007.

[9] J. L. Bona, T. Colin, and D. Lannes, "Long wave approximations for water waves," Archive for Rational Mechanics and Analysis, vol. 178, no. 3, pp. 373-410, 2005.

[10] B. Pelloni and V. A. Dougalis, "Numerical modelling of two-way propagation of non-linear dispersive waves," Mathematics and Computers in Simulation, vol. 55, no. 4-6, pp. 595-606, 2001.

[11] M. D. Buhmann, Radial Basis Functions: Theory and Implementations, vol. 12 of Cambridge Monographs on Applied and Computational Mathematics, Cambridge University Press, Cambridge, UK, 2003.

[12] C. Franke and R. Schaback, "Solving partial differential equations by collocation using radial basis functions," Applied Mathematics and Computation, vol. 93, no. 1, pp. 73-82, 1998.

[13] T. A. Driscoll and A. R. H. Heryudono, "Adaptive residual subsampling methods for radial basis function interpolation and collocation problems," Computers \& Mathematics with Applications, vol. 53, no. 6, pp. 927-939, 2007.

[14] N. Bibi, S. I. A. Tirmizi, and S. Haq, "Meshless method of lines for numerical solution of Kawahara type equations," Applied Mathematics, vol. 2, no. 5, pp. 608-618, 2011.

[15] Y. Dereli, "Solitary wave solutions of the MRLW equation using radial basis functions," Numerical Methods for Partial Differential Equations, vol. 28, no. 1, pp. 235-247, 2012.

[16] U. M. Ascher and L. R. Petzold, Computer Methods for Ordinary Differential Equations and Differential-Algebraic Equations, SIAM, Philadelphia, Pa, USA, 1998.

[17] I. Babuška, U. Banerjee, and J. E. Osborn, "Survey of meshless and generalized finite element methods: a unified approach," Acta Numerica, vol. 12, pp. 1-125, 2003.

[18] L. N. Trefethen, Spectra Methods in MATLAB, SIAM, Philadelphia, Pennsylvania, 1st edition, 2001.

[19] P. G. Drazin and R. S. Johnson, Solitons: An Introduction, Cambridge University Press, Cambridge, UK, 1st edition, 1989. 


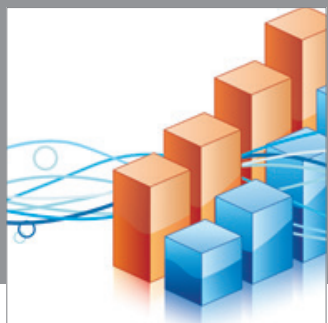

Advances in

Operations Research

mansans

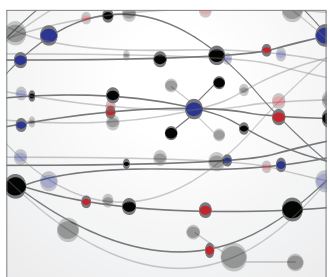

The Scientific World Journal
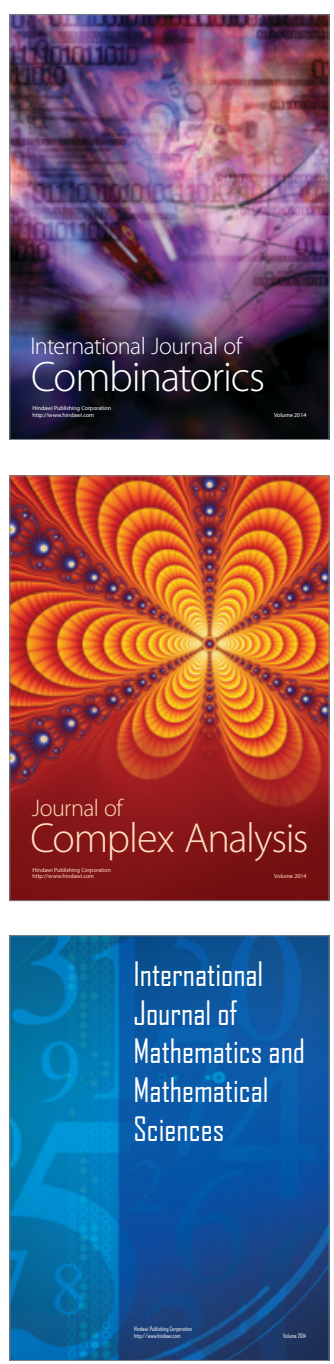
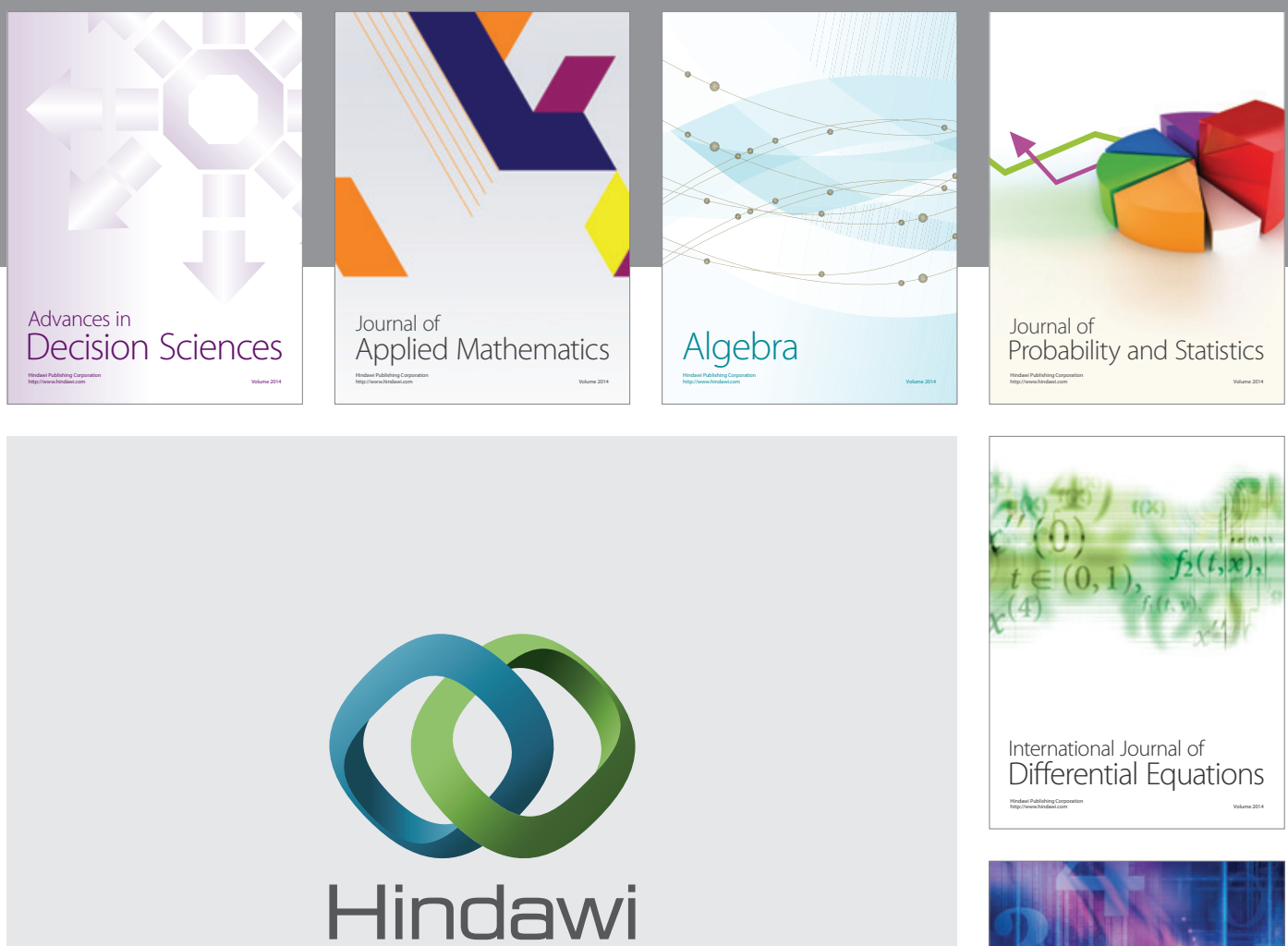

Submit your manuscripts at http://www.hindawi.com
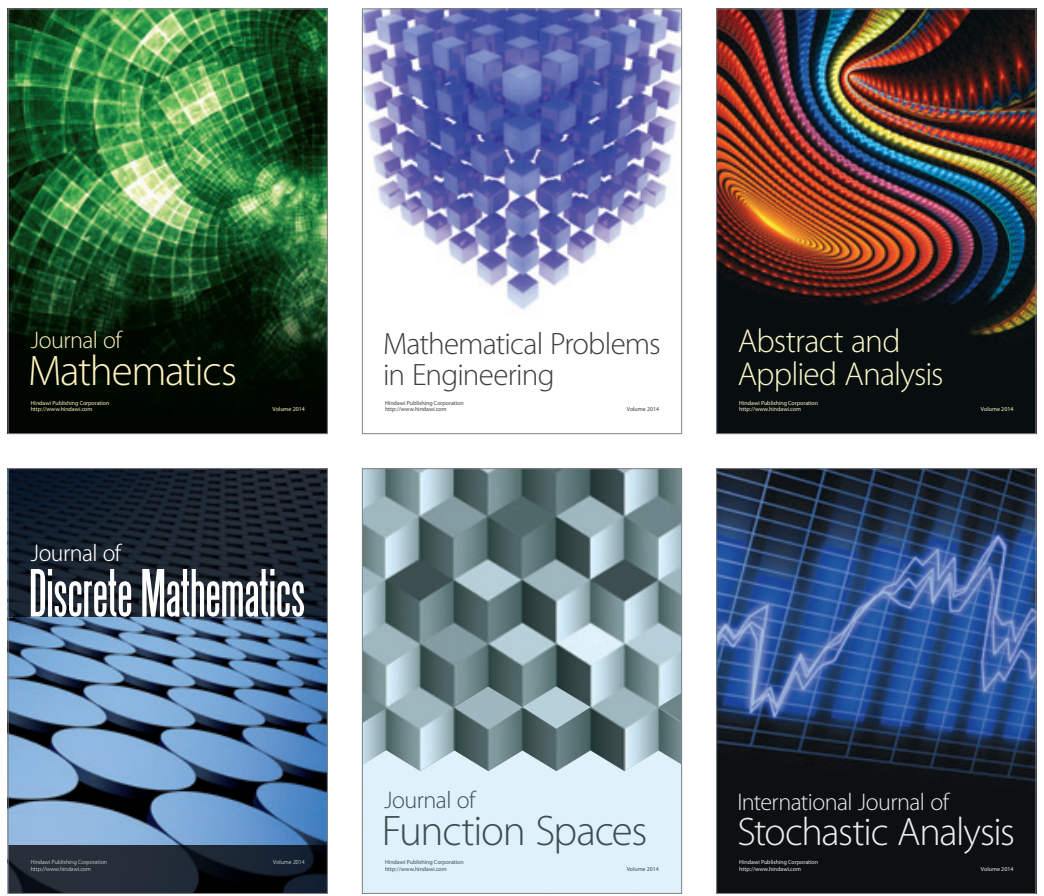

Journal of

Function Spaces

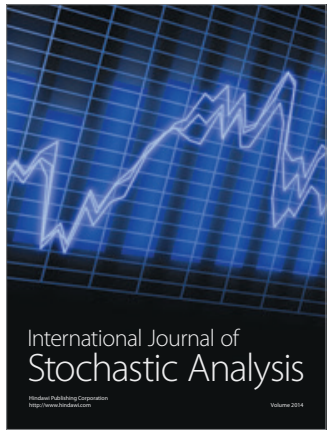

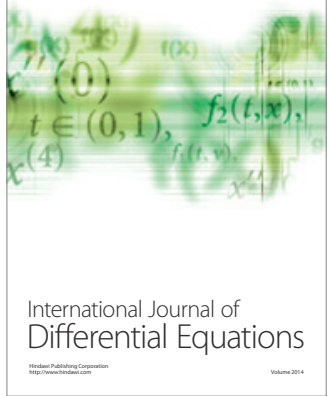
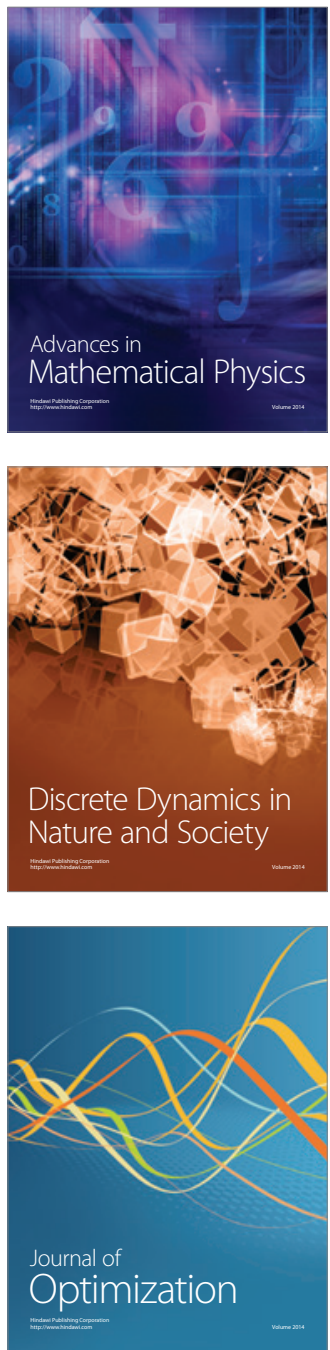